\title{
LIBERACIÓN DE OXÍGENO RADIAL POR LAS RAÍCES DE LAS PLANTAS NATIVAS DE HUMEDALES TROPICALES COSTEROS DE VERACRUZ EN RESPUESTA A DIFERENTES CONDICIONES DE INUNDACIÓN
}

\section{RADIAL OXYGEN LOSS BY ROOTS OF NATIVE TROPICAL WETLAND PLANTS OF VERACRUZ IN RESPONSE OF DIFFERENT FLOODING CONDITIONS}

\author{
Evelyn SÁnchez-Olivares ${ }^{1}$, José Luis Marín-MuñIZ Y y María Elizabeth HeRnández-Alarcón²,*
}

\begin{abstract}
${ }^{1}$ Academia de Desarrollo Regional Sustentable, El Colegio de Veracruz, Xalapa, México.
${ }^{2}$ Red de Manejo Biotecnológico de Recursos, Instituto de Ecología A.C., Xalapa, México.
\end{abstract}

*Autor de correspondencia: elizabeth.hernandez@inecol.mx

\begin{abstract}
Resumen
Antecedentes: La liberación de oxígeno por las plantas de humedales es un proceso fisiológico que oxigena el sedimento favoreciendo la actividad de los microorganismos aeróbicos, dicha actividad impacta los servicios ambientales de los humedales. Poco se sabe sobre la liberación de oxígeno por las plantas nativas de los humedales de México.

Preguntas: ¿Cuál de las macrófitas más abundantes de los humedales de Veracruz, presenta la mayor liberación de oxígeno radial? y ¿Las condiciones de inundación tienen algún efecto en la liberación de oxígeno radial de las macrófitas nativas de los humedales costeros?

Área de estudio: Veracruz, México.

Métodos: Se midió la producción y porosidad de la raíz, y la liberación de oxígeno por el método de citrato de titanio en 7 de las especies nativas de los humedales de Veracruz, crecidas bajo tres condiciones hidrológicas: capilaridad, saturación e inundación.

Resultados: Las especies con mayor peso y volumen de raíz (Pontederia sagittata, Sagitaria lancifolia y Thalia geniculata) mostraron bajas liberaciones de oxígeno radial en base a peso seco. La liberación de oxígeno por planta bajo condiciones de inundación mostró diferencias significativas entre las especies, Typha domingensis mostró mayor liberación de oxígeno $\left(148 \pm 46 \mu \mathrm{mol}_{2} \mathrm{~d}^{-1}\right)$ y Leersia ligularis la menor $\left(22 \pm 46 \mu \mathrm{mol} \mathrm{O} \mathrm{d}^{-1}\right)$.

Conclusión: La inundación ocasionó una disminución en la producción de biomasa de las raíces de las plantas nativas de los humedales de Veracruz, un aumento en la porosidad y estimuló la libración de oxígeno por planta, encontrándose diferencias significativas entre las plantas estudiadas.
\end{abstract}

Palabras clave: Aireación del sedimento, difusión de oxígeno, estrés hídrico, plantas acuáticas, porosidad de raíz.

\begin{abstract}
Background: Radial oxygen release by wetland plants is a process that creates aerobic conditions in the sediment that enhance aerobic microbial activity. Such activity has a big impact on wetland environmental services. Little is known about radial oxygen release by native macrophytes of tropical wetlands.

Study site: Veracruz, Mexico

Research Questions: Which of the most abundant native macrophytes from tropical wetlands have the higher radial oxygen release? What is the effect of hydrological conditions on radial oxygen release of the most abundant native wetlands plants of tropical wetlands?

Methods: Root production, root porosity and Oxygen radial release were measured in 7 native macrophytes of tropical wetlands in Veracruz. The macrophytes were grown under three hydrological conditions: capillarity, saturation and flooding.

Results: The species that produced more weight and volume of root (Pontederia Sagittata, Sagitaria lancifolia y Thalia geniculata) showed low radial oxygen released base on dry weight. Under flooding conditions, radial oxygen release per plant showed significant differences between the species, being Typha dominguensis the specie with the highest oxygen radial release $\left(148 \pm 46 \mu \mathrm{mol} \mathrm{O}_{2} \mathrm{~d}^{-1}\right)$ and Leersia ligularis the plant with the lowest radial oxygen release $\left(22 \pm 46 \mu \mathrm{mol} \mathrm{O}_{2} \mathrm{~d}^{-1}\right)$.

Conclusion: Flooding conditions decreased root volume and weight of native macrophytes from Veracruz wetlands, also increased root porosity and in general stimulated higher radial oxygen release per plant, with significant differences among the studied plants, indicating that radial oxygen release depend of plant phenological characteristics and the hydrological conditions.

Key words: Aquatic plants, hydric stress, oxygen diffusion, root porosity, sediment aeration.
\end{abstract}

This is an open access article distributed under the terms of the Creative Commons Attribution License CCBY-NC (4.0) international. https://creativecommons.org/licences/by-nc/4.0/ 
Los humedales son definidos como zonas de transición entre sistemas terrestres y acuáticos, donde el suelo está cubierto por una columna de agua poco profunda o el agua se encuentra a nivel del suelo, la inundación puede ser permanente o temporal, pero debe de ser suficientemente larga para permitir el desarrollo de vegetación acuática (Mitsch \& Gosselink 2015). Una parte importante de la vegetación de humedales es conocida como macrófitas (Malvárez \& Fabían 2004), y se definen como plantas acuáticas capaces de adaptarse a condiciones anaerobias y/o aerobias. Las macrófitas requieren un periodo de anegación para completar su ciclo de vida y subsisten en el agua, por lo tanto ellas pueden vivir en lugares con inundación permanente o temporal (TraviesoBello et al., 2005). Las plantas de humedales cumplen un papel importante para el ecosistema porque colaboran con la producción primaria, regulan la calidad del agua, absorben y liberan nutrientes, y diversifican los hábitats y alimento faunístico (Brix, 2004)

La inundación por largos periodos produce suelos anaerobios, es decir, suelos sin oxígeno. Las raíces de las plantas necesitan oxígeno para respirar, por lo que en un ambiente inundando como lo es un humedal, se requiere que la vegetación desarrolle varias adaptaciones (Cronk \& Fenessy 2001). Una de las adaptaciones es la modificación de la estructura de la raíz, con la producción de un tejido lleno de espacios vacíos, el cual es llamado aerénquima, y permite el transporte de oxígeno de las partes aéreas a la raíz, permitiendo la respiración de las mismas (Armstrong 1972, Keeley 1979). El oxígeno que entra por la planta a través del tejido esponjoso se difunde de las raíces hasta el suelo adyacente a esta (rizósfera), lo que se conoce como liberación radial de oxígeno (ROL por sus siglas en inglés) y a su vez provee condiciones aerobias a la zona de la rizósfera para que la raíz pueda respirar y llevar a cabo sus funciones (Armstrong 1971). La liberación de oxígeno radial tiene gran relevancia para los servicios ambientales que prestan los ecosistemas de humedales, ya que permite gradientes de óxido reducción en los sedimentos de humedales que permiten la actividad de bacterias aerobias y anaerobias en los sedimentos. La actividad de estas bacterias tiene grandes repercusiones en la depuración de agua y en el ciclamiento del carbono (Hernández 2016).

Desde hace ya varias décadas se inició el estudio de la medición de la liberación de oxígeno radial en macrófitas, mediante el establecimiento de varias técnicas, algunas utilizando métodos espectrofotométricos y otros utilizando microsensores de oxígeno (Armstrong \& Armstrong 1988, Matsui \& Tsuchiya 2006, Zhang et al. 2014, Han et al. 2016). Algunos estudios han incluido una larga lista de especies en sus estudios, encontrando diferentes tasas de ROL entre ellas, indicando que este fenómeno es característico de cada planta (Lai et al. 2012). Sin embargo, hasta la fecha, las especies estudiadas son características de humedales templados, pocas de ellas encontrados en los humedales costeros, por lo que de la mayoría de las especies de macrófitas nativas de los humedales tropicales de México, no se sabe cuál es la tasa de liberación de oxígeno radial. Los estudios sobre ROL, han indicado que la ausencia de oxígeno en el agua o suelo, in- crementa la liberación de oxígeno en algunas especies como respuesta al estrés, pero no en todos los casos (Sorrell 1999, Sasikala et al. 2009). El cambio climático está causando cambios en los hidroperiodos de los humedales costeros de México, con periodos de sequía más largos o inundaciones más profundas por las lluvias intensas (Díaz-Cordero 2012). Por lo que es pertinente estudiar cómo los cambios en los hidroperiodos de los humedales tropicales pudieran afectar las funciones fisiológicas de las macrófitas. Este estudio tuvo como objetivo cuantificar la liberación de oxígeno en las macrófitas nativas más abundantes de los humedales costeros de Veracruz, crecidas bajo diferentes hidroperiodos, simulando las condiciones de inundación en diferentes temporadas climáticas de la costa. Las preguntas de investigación que se buscaron responder fueron: ¿Cuál de las macrófitas más abundantes de los humedales de Veracruz, presenta la mayor liberación de oxígeno radial? y ¿Las condiciones de inundación tienen algún efecto en la liberación de oxígeno radial de las macrófitas nativas de los humedales costeros?

Las hipótesis planteadas fueron: a) La liberación de oxígeno radial será diferente en las macrófitas nativas de humedales tropicales, ya que ellas difieren en sus características fenológicas; b) Las condiciones de inundación y saturación ocasionarán más altas tasas de liberación de oxígeno que las condiciones de capilaridad, debido a una repuesta al estrés causado por la inundación.

\section{Materiales y métodos}

Selección y colecta de plantas. Para el estudio se seleccionaron 7 especies de plantas acuáticas emergentes (Sagittaria lancifolia L., Typha domingensis, Pontederia sagittata C. Presi, Cyperus articulatus L., Cyperus giganteus Vahl, Leersia ligularis Trin. y Thalia geniculata L.) por ser las especies más abundantes en humedales herbáceos de Veracruz, en base al valor de importancia y frecuencia descritos por Moreno-Casasola et al. (2010). En marzo de 2017, se colectaron 10 individuos adultos sin floración de cada especie, la altura de las plantas varió de acuerdo a la fenología de las mismas entre 90 y $150 \mathrm{~cm}$ de alto. Las plantas se colectaron cuidadosamente con la mano y la ayuda de una pala para no dañar la raíz. Los individuos de Sagittaria lancifolia, Typha domingensis y Pontederia sagittata se colectaron en humedales de la localidad de La Mancha Mpio. de Actopan (19 $35^{\prime} 48^{\prime}$ 'N, $\left.96^{\circ} 22^{\prime} 54^{\prime \prime} \mathrm{O}\right)$. Cyperus articulatus se colectó en un humedal de la localidad de Cansaburros en el municipio de Actopan (19 33' 09.3'’ N, 96 22' 35.3' O). En el complejo de humedales de Ciénega del Fuerte perteneciente al municipio de Tecolutla (20 $10^{\circ}$ ' 49' 'N, $96^{\circ} 55^{\prime}$ 22' 'O), se colectaron los individuos de Cyperus giganteus y Leersia ligularis. Finalmente, Thalia geniculata fue colectada en un humedal de Pinoltepec ( $19^{\circ} 26^{\prime} 45^{\prime \prime} \mathrm{N}, 96^{\circ} 45^{\prime} 18^{\prime}$ 'O), donde se habían plantado 3 años atrás individuos de esta especie que venía de humedales perilacustres de la laguna de San Julián $\left(19^{\circ} 15^{\prime} 27.7^{\prime}\right.$ 'N, $96^{\circ} 15^{\prime} 47.8^{\prime}$ 'O), Mpio. del puerto de Veracruz.

Las plantas se transportaron sumergidas en agua a las instalaciones del Instituto de Ecología, A.C. en la Ciudad 
de Xalapa (19 $32^{\prime} 31^{\prime \prime}$ N, 95 54'35'O), en donde se llevaron a cabo su propagación y la medición de la liberación de oxígeno.

Propagación y crecimiento de las plantas. La propagación y el crecimiento de las plantas se llevó a cabo en un invernadero rustico en el campus III del Instituto de Ecología A.C. en la Ciudad de Xalapa, Ver. El invernadero tiene una cubierta de plástico de una altura de $3 \mathrm{~m}$ en forma de túnel, las paredes del invernadero $(1.5 \mathrm{~m})$ de alto están cubiertas por malla mosquitera. En el invernadero no había control de temperatura ni humedad relativa. La temperatura ambiente dentro del invernadero se registró diariamente y la media durante la propagación y crecimiento de las plantas fue de $23{ }^{\circ} \mathrm{C}$ y el fotoperiodo de luz de día y obscuridad durante la noche. Cinco individuos de cada especie se sembraron en contenedores de plástico de $60 \times 40 \times 34 \mathrm{~cm}$, que contenían una columna de suelo de $40 \mathrm{~cm}$, el cual se inundó completamente con agua de la llave El suelo provenía de los primeros $30 \mathrm{~cm}$ de los humedales herbáceos de Ciénega del Fuerte municipio de Tecolutla $\left(20^{\circ} 18^{\prime} 49^{\prime}\right.$ ' $\mathrm{N}, 96^{\circ} 55^{\prime}$ 22 ' $\mathrm{O}$ ), este sitio fue elegido por cuestiones logísticas de apoyo de campo para la colecta del suelo y el permiso de los locales para recolectarlo. La composición química del suelo se caracterizó para otro proyecto (Cruz-Zamora 2018) y tenía la siguiente composición: $\mathrm{C}(21 \%) \mathrm{N}(1.8 \%), \mathrm{PO}_{4}$ (0.9 mg/kg), $\mathrm{NO}_{3}(10 \mathrm{mg} / \mathrm{kg}), \mathrm{pH}$ (7.50). Se utilizó suelo de humedales para tener las plantas en condiciones lo más similares a los ecosistemas en el que crecen. A pesar de ser este suelo rico en materia orgánica y nutrientes se decidió fertilizar las tinas de las plantas madres, con el fin de acelerar la aparición de rebrotes y asegurar el crecimiento de las plantas en 20 días para la medición de la liberación de oxígeno por las raíces. Todas las plantas se mantuvieron bajo las mismas condiciones de suelo y nutrientes independientemente del lugar en donde fueron colectadas, lo anterior, con el fin de estandarizar las condiciones de propagación y crecimiento, para que la única variable fuese las condiciones de inundación.

Cada semana se añadía 1. $5 \mathrm{~L}$ de solución de nutrientes preparada con el fertilizante comercial Triple 17 marca "nutrigarden", que contenía N, P y K a una concentración de 5 $\mathrm{mg} \mathrm{L}^{-1}$. Una vez obtenidos los rebrotes de cada especie (entre 10 y 15 días), se sembró un individuo (de entre 20 y $30 \mathrm{~cm}$ de altura, media $24.2 \mathrm{~cm})$ en bolsas de plástico negro $(13 \times$ $25 \mathrm{~cm}$ ) con suelo de humedal del mismo lote del puesto en las tinas con las plantas madre. A las bolsas se les hicieron 10 agujeros de $2 \mathrm{~cm}$ para permitir el contacto con el agua. Las bolsas con suelo y plantas se colocaron en contenedores de plástico de $60 \times 40 \times 34 \mathrm{~cm}$ que contenían agua. Se colocaron 8 plantas por cada especie en cada contenedor bajo tres diferentes condiciones hídricas ( 24 plantas en total por cada especie). Las condiciones hídricas fueron: capilaridad (las bolsas estaban sumergidas $10 \mathrm{~cm}$ ), saturación (las bolsas estaban sumergidas $30 \mathrm{~cm}$ hasta la base de la planta) e inundación (las bolsas estaban sumergidas $40 \mathrm{~cm}$, con una columna de agua de $10 \mathrm{~cm}$ arriba de la base de la planta). Los contenedores que contenían las plantas se enriquecieron al día cero y el día 14 con fertilizante Triple $17\left(5 \mathrm{mg} \mathrm{L}^{-1}\right.$ de NPK), en los tres tipos de inundación.

Las plantas se dejaron crecer bajo las diferentes condiciones de inundación durante 20 días, se midió su altura al inicio y al día 20. Después, se sacaron de las bolsas y se retiró la tierra adherida a las raíces, se lavaron para eliminar materia extraña y se pusieron en solución nutritiva (fertilizante triple 17 a una concentración de $5 \mathrm{mg} \mathrm{L}^{-1}$ ) en frascos de $212 \mathrm{ml}$, con tapón de rosca que tenía un orificio por donde la parte área de la planta se sacó del frasco, solo la raíz estuvo completamente sumergida en la solución. Este tapón también tenía un septo de goma (utiryl gris) para extraer o inyectar solución en el frasco sin necesidad de abrirlo. La base de la planta se fijó al tapón de plástico con parafina, para que quedara fija y se evitará la entrada de aire al frasco. Las plantas se dejaron en la solución nutritiva por 24 horas antes de realizar la medición de liberación de oxígeno, con el fin de que cualquier traza de suelo adherida a la raíz se quedará en la solución nutritiva y las plantas se aclimataran a la solución antes de la medición. Se utilizaron 5 réplicas por cada especie en cada condición de inundación para la medición de liberación de oxígeno. Las plantas sobrantes de las 8 crecidas en cada condición de inundación se utilizaron para medir la porosidad de la raíz.

Medición de liberación de oxígeno. La tasa de liberación de oxígeno se determinó de acuerdo con el método de citrato de titanio $\left(\mathrm{Ti}^{3}+\right)$ descrito por Kludze et al. (1994) y Mei et al. (2009). La preparación de la solución de citrato de titanio se realizó bajo condiciones reducidas en una cámara libre de oxígeno. Se añadieron $270 \mathrm{ml}$ de agua destilada previamente burbujeada con $\mathrm{N}_{2}$ a 15.88 gr de citrato de sodio para dar solución a citrato sódico $0.2 \mathrm{M}$. Después se agregaron $27 \mathrm{ml}$ de cloruro de titanio (Aldrich Chemical Co.) a la solución de citrato sódico, se ajustó la solución a un $\mathrm{pH}=5$ con carbonato de sodio saturado ( 25 gr de $\mathrm{NaCO}_{3}$ y $100 \mathrm{ml}$ de agua).

Se preparó solución nutritiva (fertilizante triple 17 a una concentración de $5 \mathrm{mg} \mathrm{L}^{-1}$ ) y se colocaron $170 \mathrm{ml}$ en frascos con tapón de rosca hermético con un volumen total de $212 \mathrm{ml}$ los cuales se burbujearon con $\mathrm{N}_{2}$ durante 2 min para eliminar el $\mathrm{O}_{2}$ disuelto. Los frascos herméticamente cerrados, se colocaron dentro de la cámara libre de oxígeno y allí se cambió el tapón del frasco por el tapón con la planta fijada con parafina que había estado en solución nutritiva por $24 \mathrm{~h}$. La raíz de la planta quedó sumergida en la solución nutritiva libre de oxígeno. Los frascos con planta sellados herméticamente se sacaron de la cámara y a través del septo se inyectaron $20 \mathrm{ml}$ de la solución de citrato de titanio y se agitaron manualmente con cuidado de no dañar la parte aérea de las plantas. Inmediatamente después de la agitación, se tomó a través de septo una muestra de $2 \mathrm{ml}$ de la solución nutritiva con citrato de sodio y se colocó en un vial previamente evacuado al vacío y sellado herméticamente. Las plantas se colocaron en una mesa a temperatura ambiente donde les llegaba luz natural a través de una ventana en el laboratorio y a las seis horas se volvió a tomar otra muestra de la solución nutritiva con citrato de sodio. La absorbancia de las muestras de citrato de titanio tomadas a diferentes tiempos se midió a $527 \mathrm{~nm}$ en 
un espectrofotómetro Jenway UV. La cantidad de $\mathrm{O}_{2}$ en las muestras de citrato de titanio se calculó a partir de una curva de calibración que se realizó inyectando concentraciones conocidas de oxígeno a una solución de citrato de Titanio $0.1 \mathrm{M}$. En cada medición se utilizaron 5 réplicas para cada planta y tipo de inundación, y un control, que no contenía plantas. Después de medir el ROL, las plantas se sacaron de la solución de citrato de titanio, se lavaron y se les midió el volumen de raíz, posteriormente se dividieron entre biomasa aérea y raíz y ambas partes se pusieron a secar para calcular el peso seco. Para calcular la liberación de oxígeno (ROL) por las raíces se utilizó la siguiente formula:

$$
R O L=((y-z) / t) \times 24
$$

donde $R O L=$ liberación de oxígeno, $\mu$ mol $\mathrm{O}_{2}$ planta $^{-1}$ día $^{-1}$; $z=$ concentración de oxígeno en la solución de citrato de titanio con planta después de $6 \mathrm{~h}$ de tratamiento con planta, $\mu \mathrm{mol} \mathrm{O}_{2} ; y=$ concentración inicial de oxígeno en la solución de citrato de titanio en la solución con planta, $\mu \mathrm{mol} \mathrm{O}_{2}, t=$ tiempo del experimento $(6 \mathrm{~h})$ y $24=$ factor de conversión de horas por día.

Medición de la porosidad. A tres individuos de cada especie, crecidas bajo las condiciones de inundación, se les midió la porosidad de la raíz, mediante el método descrito por Van-Noordwijk \& Brouwer (1988). Las muestras de raíces se cortaron en trozos de $5 \mathrm{~cm}$ de longitud, con un total de 3 gr por cada muestra. La primera medida que se tomó fue el peso del picnómetro lleno con agua. La segunda medida fue la del picnómetro lleno con los $3 \mathrm{~g}$ de raíz aforados con agua. Después se retiraron las raíces del picnómetro y se secaron delicadamente con papel absorbente con el objetivo de retirar el agua adherida y se pesaron las raíces; posteriormente para retirar el agua absorbida se molieron las raíces en mortero y se introdujeron al picnómetro con agua, y se pesó el picnómetro. Cabe señalar que antes de cada medición el picnómetro se introdujo en un baño de agua para mantener la temperatura a $20^{\circ} \mathrm{C}$. La fórmula para calcular la porosidad es la siguiente:

$$
P O R=\left(\left(P R^{\prime}-P R\right) /((\mathrm{a}+\mathrm{P})-\mathrm{PR})\right) \times 100
$$

donde:

$P O R=$ Porosidad de la raíz $\%$.

$P R=$ Peso picnómetro con raíces con poros llenos de aire y agua, g.

$P R^{\prime}=$ Peso picnómetro con raíces sin poros llenos de aire y agua, $g$.

$P=$ Peso picnómetro lleno de agua, $\mathrm{g}$.

$a=$ Peso fresco de las raíces, g.

Medición de volumen de raíces. El volumen de raíz se midió por el método de desplazamiento del volumen de agua. Primero se eligió un frasco cuyo volumen era de $212 \mathrm{ml}$, en él se colocaban las raíces de las plantas de tal modo que el final del tallo quedara sobre el borde del frasco, después en un vaso de precipitados se colocaban los $212 \mathrm{ml}$ de agua, y poco a poco se introducía el agua al frasco donde estaban las raíces hasta que el agua rebosara, sin que se tirara líquido, finalmente se medía el agua sobrante, la cual era el volumen de raíz.

Medición de peso seco. Se cortaron las plantas en dos partes, la parte aérea que contempla los tallos y las hojas, y la otra parte son las raíces. Ambas partes se colocan en papel y se introducen en una estufa a $105^{\circ} \mathrm{C}$ de laboratorio hasta peso constante.

Análisis estadísticos. Los análisis estadísticos fueron realizados con PAST (Paleontogical Statistics Software Package for Education and Data Analysis) versión 3.16 para Windows. Se realizó el análisis de normalidad a los datos con la prueba Levene's test. Para los datos que fueron normarlos se aplicó un análisis de varianza (ANOVA) de una vía para evaluar si había un efecto en la liberación de oxígeno por gramo de raíz, liberación de oxígeno por planta, porosidad, y peso seco de raíz de las plantas de humedal de acuerdo a su nivel de inundación. La prueba poshoc de tukey's se utilizó para detectar diferencias significativas entre los tratamientos. Si los datos no eran normales, entonces realizaba una prueba KruskalWallis. Un valor de $p \leq 0.05$ fue utilizado para revelar la significancia estadística en todas las estimaciones.

\section{Resultados}

Producción de raíz en peso y volumen. Bajo condiciones de inundación, L. ligularis, produjo significativamente $(p<0.05)$ menor peso seco $(0.175 \mathrm{~g})$ que $P$. sagittata, S. lancifolia, T. geniculata, C. giganteus y T. domingensis (Figura 1A). Sin embargo, no fue significativamente $(p=0.0627)$ diferente del peso seco de raíz de $C$. articulatus $(0.326 \mathrm{~g})$ y este fue significativamente $(p \leq 0.05)$ menor del peso seco de $T$. geniculata $(1.52 \mathrm{~g})$ y $T$. domingensis $(0.88 \mathrm{~g})$. En la condición de saturación $L$. ligularis tuvo significativamente $(p<0.05)$ menor peso seco de raíz $(0.25 \mathrm{~g})$ que $P$. sagittata (0.92 g), T. geniculata $(1.16 \mathrm{~g})$ y C. giganteus $(0.81 \mathrm{~g})$.

En capilaridad, $P$. sagittata y $S$. lancifolia produjeron significativamente $(p<0.05)$ mayor peso seco de raíz $(1.6$ y 1.7 g respectivamente) que $C$. articulatus, L. ligularis, $C$. giganteus y $T$. domingensis. Sin embargo, no fueron estadísticamente significativas $(p>0.05)$ del peso seco de raíz de T. geniculata $(1.28 \mathrm{~g})$.

El volumen de la raíz tuvo un comportamiento similar al peso seco (Figura 1B). Bajo condiciones de inundación $P$. sagittata $(19.4 \pm 2.56 \mathrm{ml})$, S. lancifolia $(15.2 \pm 1.9 \mathrm{ml}), T$. geniculata $(16.4 \pm 2.9 \mathrm{ml})$ tuvieron significativamente $(p<$ $0.05)$ mayor volumen de raíz que $C$. articulatus $(8.8 \pm 0.4$ $\mathrm{ml})$ y L. ligularis $(3.2 \pm 1.2 \mathrm{ml})$. A su vez C. giganteus $(12$ $\pm 3.6 \mathrm{ml}), T$. domingensis $(16.5 \pm 4.3 \mathrm{ml})$ y $C$. articulatus $(8.8 \pm 0.4 \mathrm{ml})$ tuvieron mayor volumen de raíz con respecto L. ligularis, pero no significativamente mayor que $C$. articulatus. En condiciones de saturación, P. sagittata (20.6 \pm $3.4 \mathrm{ml})$, S. lancifolia $(24.8 \pm 6.4 \mathrm{ml})$, T. geniculata $(15.2 \pm$ $0.8 \mathrm{ml})$ y $T$. domingensis $(13 \pm 2.7 \mathrm{ml})$ tuvieron significativamente $(p<0.05)$ mayor volumen de raíz que L. ligularis $(4.7 \pm 1.8 \mathrm{ml})$. También se observó que $P$. sagittata generó 


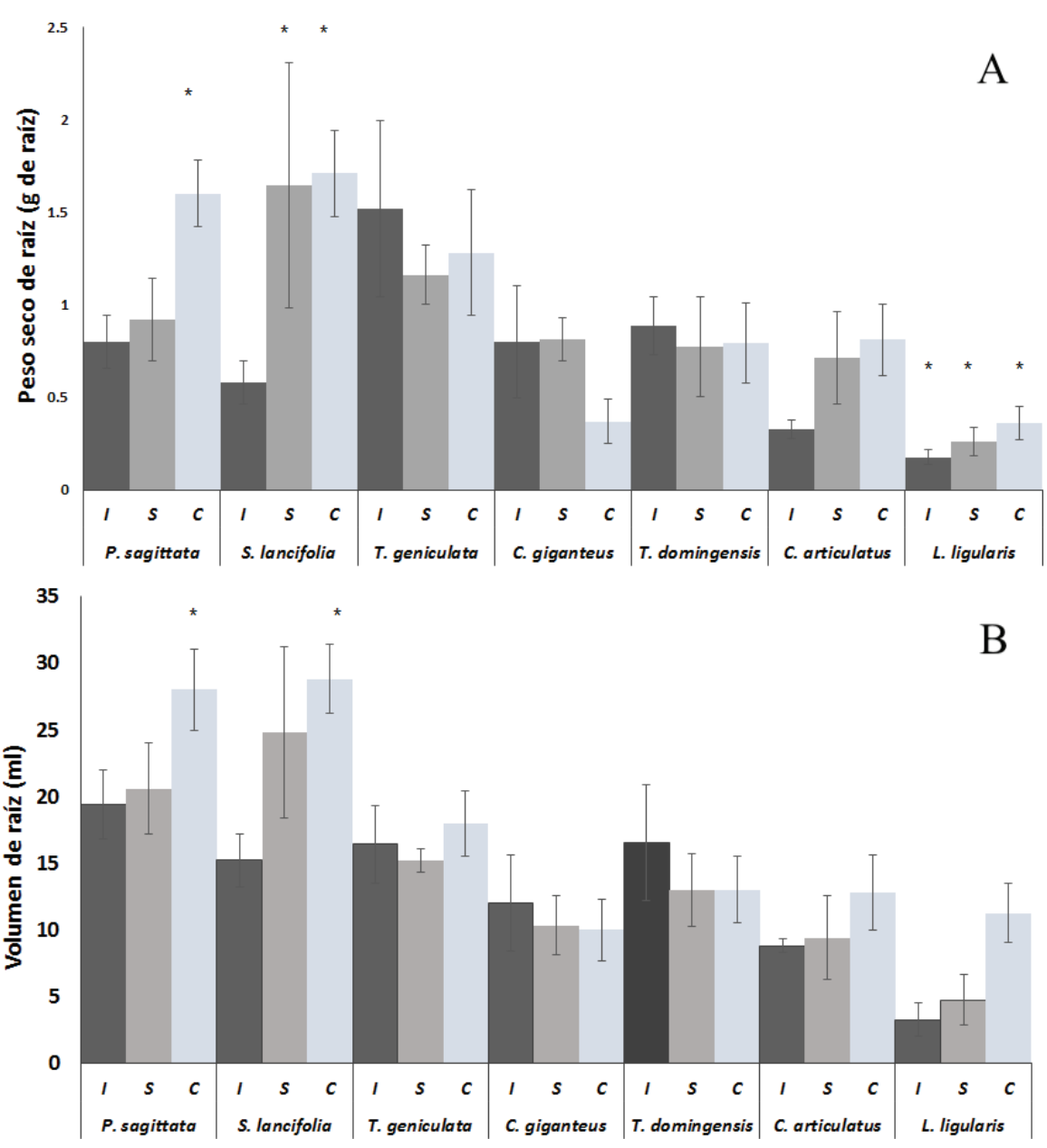

Figura 1. Producción de raíz con base en peso seco (A) y volumen (B) de las macrófitas más abundantes de los humedales costeros de Veracruz, crecidas bajo diferentes condiciones de inundación. I = Inundación, $\mathrm{S}=$ Saturación, $\mathrm{C}=$ capilaridad. Los valores son promedios ( $\mathrm{n}=5$ ), las barras representan el error estándar. * indica diferencias significativas.

significativamente $(p=0.0353)$ mayor volumen de raíz que C. articulatus $(9.4 \pm 3.1 \mathrm{ml})$, y C. giganteus $(10.3 \pm 2 \mathrm{ml})$ que T. geniculata $\operatorname{con} p=0.0480$. Por otra parte, P. sagittata $(28$ $\pm 3.8 \mathrm{ml})$, S. lancifolia $(28.8 \pm 2.5 \mathrm{ml})$ mostraron significativamente $(p<0.05)$ mayor volumen de raíz que $T$. geniculata $(18 \pm 2.4 \mathrm{ml}), C$. giganteus $(10 \pm 2.3 \mathrm{ml}), T$. domingensis $(13 \pm 2.4 \mathrm{ml})$, C. articulatus $(12.8 \pm 2.8 \mathrm{ml})$ y L. ligularis $(11.25 \pm 2.21 \mathrm{ml})$.

Porosidad de las raices. Para la porosidad, se encontraron patrones diferentes a los de producción de raíz, especies con alta producción de raíz, como $P$. sagittata y $T$. geniculata, presentaron porosidades entre 20 y $30 \%$, mientras especies con producción intermedia de raíz como $C$. giganteus $T$. domingensis y $C$. articulatus presentaron porosidades entre $37-57 \%$ (Figura 2). L. ligularis al igual que el peso seco, fue la especie que presentó la porosidad más baja $(19.9 \pm 2.9 \%)$.

Liberación de oxígeno por gramo de raíz. Excepto para $C$. gigantus y L ligularis, la liberación de oxígeno por las raíces de las plantas de humedales mostraron mayores valores en condicones de inundación y saturación, sin embargo las diferencias no fueron estadisticamente significativas $(p>0.05)($ Figura 3 ). Las especies con mayores valores de liberación de oxígeno fueron $T$. domingensis, $C$. articulatus y L. ligularis con valores en el rango de 140 a $180 \mu \mathrm{mol} \mathrm{O}_{2} \mathrm{~g}^{-1}$ y especies como $P$. sagitatta, $S$ lancifolia y $T$. geniculata, mostarron menores valores de liberación de oxígeno en el rango de 40 a $100 \mu \mathrm{mol} \mathrm{O} \mathrm{g}^{-1} \mathrm{~d}^{-1}$.

Liberación de oxígeno por planta. Cuando analizamos la liberación de oxígeno sin tomar en cuenta el peso de la raíz, esto es la liberación de oxígeno por planta (Figura 4) observamos que $T$. geniculata, $T$. dominguensis y $C$. articulatus mostraron mayor liberación de oxígeno en condiciones de inundación que en capilaridad, sin embrago las diferencias no fueron estadísticamente significativas $(p<0.05)$. El valor más alto de liberación de oxígeno $(148 \pm 46 \mu \mathrm{mol}$ $\mathrm{O}_{2} \mathrm{~d}^{-1}$ ) se observó para $T$. dominguensis en condiciones de inundación y fue significativamente más alto $(p<0.05)$ que 


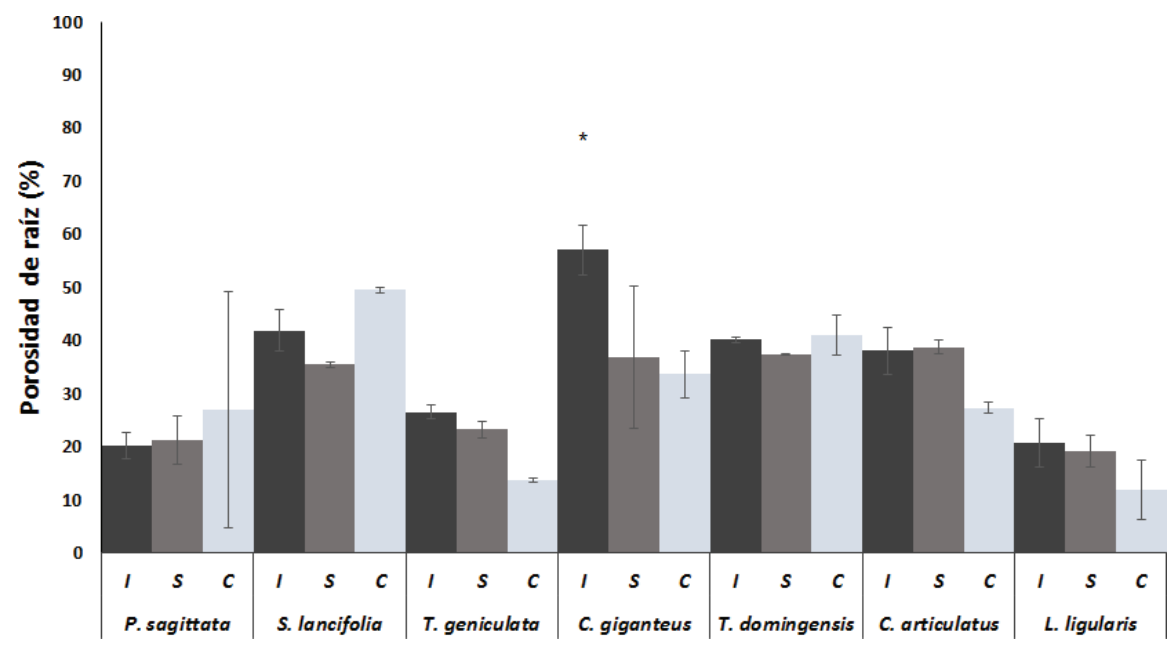

Figura 2. Porosidad de las raíces las macrófitas más abundantes de los humedales costeros de Veracruz, crecidas bajo diferentes condiciones de inundación. I = Inundación, $\mathrm{S}=$ Saturación, $\mathrm{C}=$ capilaridad. Valores son promedios $(\mathrm{n}=5)$, barras representan el error estándar. * indica diferencias significativas.

los valores más bajos observados en L. ligularis $(22 \pm 46$ $\left.\mu \mathrm{mol} \mathrm{O}_{2} \mathrm{~d}^{-1}\right)$ y $S$. lancifolia, $\left(37 \pm 12 \mu \mathrm{mol} \mathrm{O}_{2} \mathrm{~d}^{-1}\right)$, en las otras dos condiciones de inundación, no hubo diferencias significativas $(p \geq 0.05)$ entre las distintas especies estudiadas.

\section{Discusión}

En este estudio se observó que para algunas especies nativas de los humedales tropicales de Veracruz, la producción de raíz se ve disminuida bajo condiciones de inundación, esto se debe a una respuesta al estrés hídrico (Jackson \& Colmer 2005). Esto coincide con los resultados observados por Sasikala et al. (2009), quienes observaron en mesocosmos de humedales que Thypha orientalis produjo menos peso de raíz en condiciones permanentemente inundados en comparación con plantas crecidas con inundación intermitente. Se encontró que especies con mayor producción de biomasa de raíz ( $P$. sagittata, $S$. lancifolia y $T$. geniculata), fueron las especies con menor liberación de oxígeno. Estos resultados concuerdan con lo observado por Sorrell (1999), quien encontró que $J$. inflexus produjo mayor peso seco de raíz que $J$. effusus y la liberación de oxígeno fue menor en la primera especie. Los autores explican que la liberación de oxígeno no sólo está relacionada con el peso seco de la raíz si no con la morfología de la raíz. Sin embrago, otros estudios han encontrado una correlación positiva entre el peso seco de la raíz y la liberación de oxígeno (Lai et al. 2012).

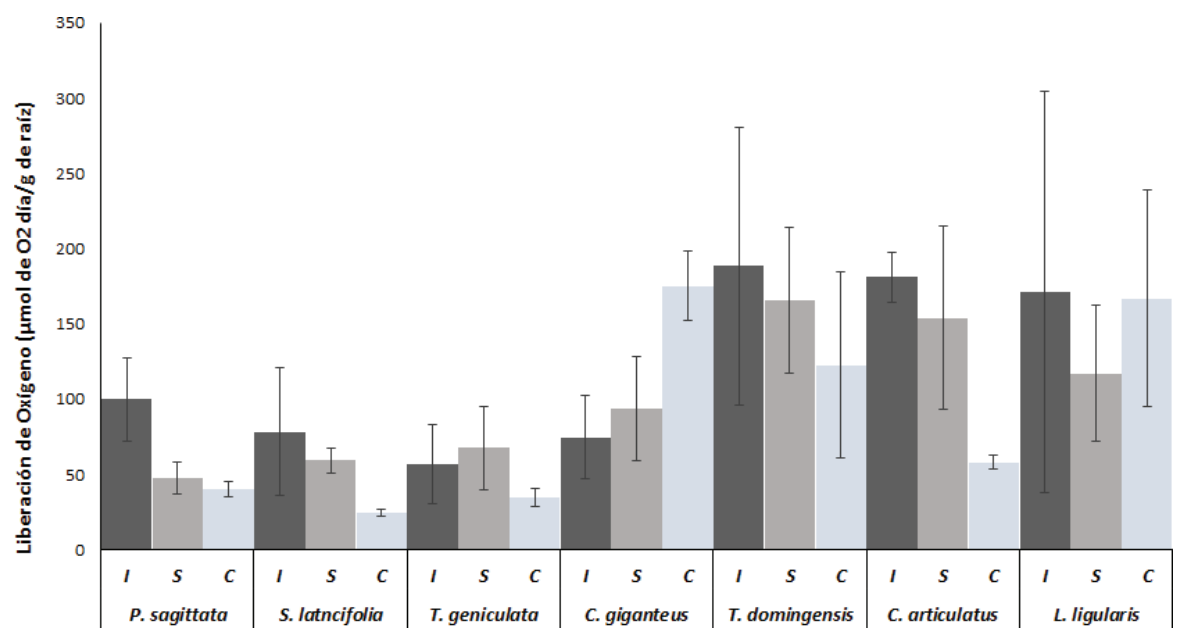

Figura 3. Liberación de oxígeno radial en base a peso seco de las raíces de las macrófitas más abundantes de los humedales costeros de Veracruz, crecidas bajo diferentes condiciones de inundación. $\mathrm{I}=$ Inundación, $\mathrm{S}=$ Saturación, $\mathrm{C}=$ capilaridad. Valores son promedios $(\mathrm{n}=5)$, barras representan el error estándar. * indica diferencias significativas 


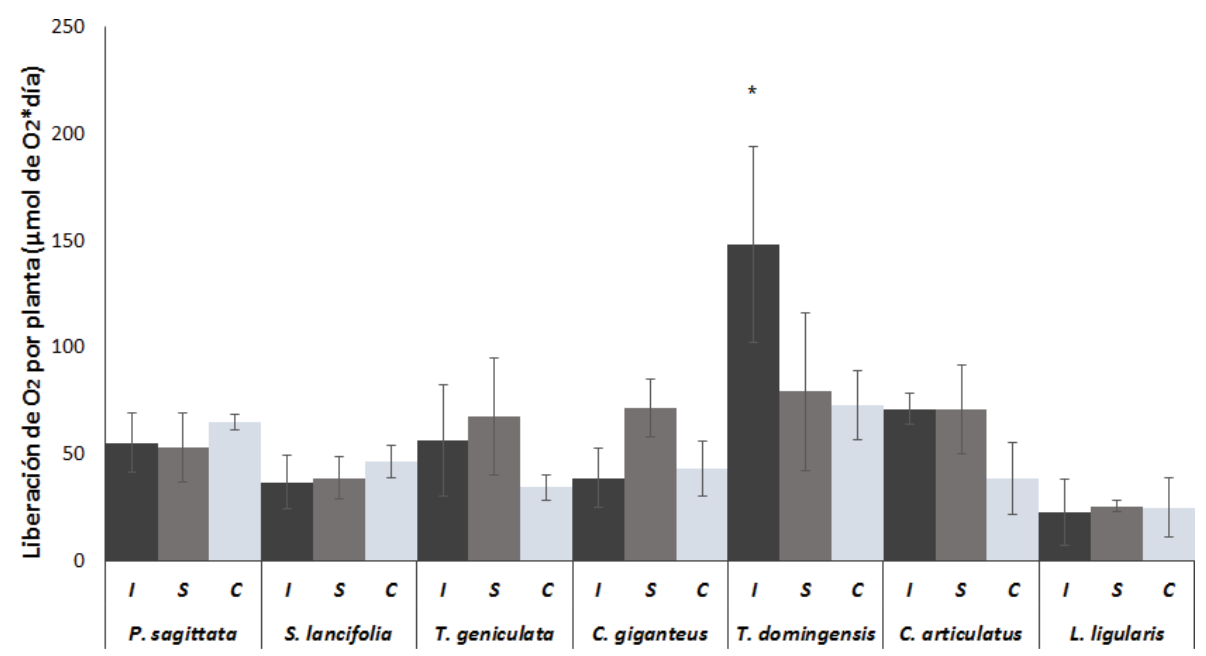

Figura 4. Liberación de oxígeno radial por planta de las raíces de las macrófitas más abundantes de los humedales costeros de Veracruz, crecidas bajo diferentes condiciones de inundación. $\mathrm{I}=$ Inundación, $\mathrm{S}=$ Saturación, $\mathrm{C}=$ capilaridad. Valores son promedios $(\mathrm{n}=5)$, barras representan el error estándar. * indica diferencias significativas.

En este estudio sólo observamos diferencias significativas entre la liberación de oxígeno por planta bajo condiciones de inundación, por lo que aceptamos la hipótesis a). Sin embargo, para las condiciones de saturación y capilaridad no observamos diferencias significativas entre las especies, por lo que para estas condiciones rechazamos la hipótesis a). Lo anterior pudiera explicarse porque bajo la condición de capilaridad hay más disponibilidad de oxígeno en el sedimento y bajo condiciones de saturación, aunque más limitado aún pudiera haber cierta difusión de oxígeno de la atmósfera al sedimento, por lo que bajo dichas condiciones las especies de macrófitas estudiadas están bien habilitadas para transportar

Tabla 1. Comparación de la liberación de oxígeno por varias plantas de humedales

\begin{tabular}{|c|c|c|c|c|c|}
\hline Especie & $\begin{array}{c}\text { Liberación de Oxígeno } \\
\left(\mu \mathrm{mol} \mathrm{O}_{2} \mathrm{~d}^{-1} \mathrm{~g}^{-1} \text { seco de raíz }\right)\end{array}$ & $\begin{array}{c}\text { Liberación de } \\
\text { Oxígeno } \\
\left(\mu \mathrm{mol} \mathrm{O} \mathrm{O}_{2} \mathrm{~d}^{-1)}\right.\end{array}$ & $\begin{array}{l}\text { Porosidad de la } \\
\text { raíz (\%) }\end{array}$ & $\begin{array}{l}\text { Peso seco de raíz } \\
\text { (g base seca) }\end{array}$ & Referencia \\
\hline Juncus effusus & 348 & 386.4 & 39 & 0.11 & Sorrell 1999 \\
\hline Juncus ingens & 348 & NR & NR & NR & Sorrell 1999 \\
\hline Juncus inflexus & 194 & 163 & 51 & 0.203 & Sorrell 1999 \\
\hline Juncus bulbosus & 36 & NR & & & Chabbi 1999 \\
\hline Cladium sp & 40.8 & NR & $9-15$ & NR & Chabbi et al. 2000 \\
\hline Typha $\mathrm{sp}$ & 45.6 & NR & $10-28$ & NR & $\begin{array}{l}\text { Chabbi et al. } 2000 \\
\text { Mei et al. } 2009\end{array}$ \\
\hline Typha orientalis & $60-180$ & NR & $30-40$ & $3.14-12.56$ & Sasikala et al. 2009 \\
\hline Potamogeton coloratus & 36 & NR & $10-20$ & NR & Lemoine et al. 2012 \\
\hline Sparganium emersum & 43 & NR & $12-18$ & NR & Lemoine et al. 2012 \\
\hline Elodea canadensis & 60 & NR & $12-18$ & NR & Lemoine et al. 2012 \\
\hline Thalia geniculata & $34-56$ & $34-67$ & $15-28$ & $0.6-1.6$ & Este estudio \\
\hline Sagitaria lancifolia & $23-160$ & $38-46$ & $42-50$ & $0.5-1.5$ & Este estudio \\
\hline Thypa domingensis & $122-188$ & $72-148$ & $35-40$ & $0.7-0.9$ & Este estudio \\
\hline Cyperus giganteus & $74-175$ & $39-71$ & $38-55$ & $0.4-0.8$ & Este estudio \\
\hline Pontederia sagittata & $39-99$ & $53-65$ & $20-24$ & $0.6-1.5$ & Este estudio \\
\hline Cyperus articulatus & $58-181$ & $38-71$ & $25-34$ & $0.3-0.7$ & Este estudio \\
\hline Leersia ligularis & $117-171$ & $22-25$ & $12-19$ & $0.1-0.7$ & Este estudio \\
\hline
\end{tabular}

$\mathrm{NR}=$ No reportado 
el poco oxígeno que haga falta sin maximizar su capacidad como lo tuvieron que hacer bajo condiciones de inundación. Se ha reportado que cuando el sedimento está seco disminuye la producción de aerénquima, que es el tejido por el que se transporta el oxígeno de las partes aéreas a la raíz (Sorrell 1999). Lo anterior pudiera explicar la respuesta observada en algunas especies de disminución de la liberación de oxígeno por la raíz bajo la condición de capilaridad.

Con excepción de $P$. sagittata y $S$. lancifolia, las demás especies estudiadas mostraron una tendencia a aumentar su porosidad bajo condiciones de inundación; esto ha sido observado en otras especies nativas de humedales templados (Sasikala et al. 2009). Las porosidades encontradas en este estudio estuvieron en el rango de 19 a $57 \%$, las cuales se encuentran dentro del rango reportado para otras especies de plantas acuáticas (Tabla 1). Los valores de liberación de oxígeno radial en base a peso seco, observados en este estudio estuvieron en el rango de 24 a $188 \mu \mathrm{mol} \mathrm{O}_{2} \mathrm{~g}^{-1} \mathrm{~d}^{-1}$, los cuales se compararon con los reportados en la literatura para otras plantas nativas de humedales templados utilizando el mismo método de citrato de Ti (Tabla 1). En este estudio encontramos que Typha dominguensis presentó la mayor liberación de oxigeno radial por planta, en comparación con los otros géneros estudiados. Debido a la amplia distribución geográfica del género Typha, este ha sido uno de los más estudiados en cuanto la liberación de oxígeno radial (Matsui \& Tsuchiya 2006, Matsui \& Tsuchiya 2008, Sasikala et al. 2009). En otros estudios donde se comparó la liberación de oxígeno por las raíces de Typha con otras plantas como Juncus effusus y Cladium jamicense, también se encontró que Typha presento mayor liberación de oxígeno por las raíces (Weissner et al, 2002, Chabbi et al. 2000). Lo anterior fue explicado por los autores, debido al mayor contenido de tejido de aerénquima en Typha, en comparación con las otras plantas.

\section{Agradecimientos}

El financiamiento para este Proyecto fue proporcionado por el Consejo Nacional de Ciencia y Tecnología, a través de la beca No. 782402 otorgada al primer autor y el Fondo de Ciencia Básica a través del proyecto 258412.

\section{Literatura citada}

Armstrong W. 1971. Radial oxygen losses from intact rice roots as affected by distances for the apex, respiration and waterlogging. Physiologia Plantarum 7: 192-197. DOI: https://doi.org/10.1111/j.1399-3054.1971.tb01427.x

Armstrong W. 1972. A Re-examination of the functional significance of aerenchyma. Physiologia Plantarum 27: 173-177. DOI: https://doi.org/10.1111/j.1399-3054.1972.tb03596.x

Armstrong W, Armstrong J. 1988. Phragmites australis -a preliminary study of soil- oxidizing sites and internal gas transport pathways. New phytologist 108: 373-382. DOI: https://doi.org/10.1111/j.1469-8137.1988.tb04177.x

Brix H. 1994. Functions of Macrophytes in Constructed Wetlands. Water Science and Technology 29: 71-78. DOI: https://doi.org/10.2166/wst.1994.0160
Chabbi A. 1999. Juncus bulbosus as a pioneer species in acidic lignite mining lakes: interactions, mechanism and survival strategies. New Phytologyst 144: 133-142. DOI: https://doi.org/10.1046/j.1469-8137.1999.00503.x

Chabbi A, McKee KL, Mendelssohn IA. 2000. Fate of oxygen losses from Typha domingensis (Typhaceae) and Cladium jamaicense (Cyperacea) andconsequences for root metabolism. American Journal of Botany 87: 1081-1090. DOI: https://doi.org/10.2307/2656644

Cronk JK, Fennesy MS. 2001. Wetland Plants: Biology and Ecology. Boca Raton, FL.: Lewis Publishers. ISBN: 9781566703727

Cruz-Zamora ED. 2018. Caracterización fisicoquímica de de humedales naturales y perturbados. Tesis de licenciatura. Facultad de Ingeniería Ambiental. Instituto Tecnológico de Álamo Temapache. Xoyotitla, Mpio. de Álamo Temapache, Ver., 48 pp.

Díaz-Cordero G. 2012. El Cambio Climático. Ciencia y Sociedad 37: 227-240.

Han C, Ren J, Tang H, Xu D, Xie X. 2016. Quantitative imaging of radial oxygen loss from Valisneria spiralis roots with a fluorescent planar optode. Science of the Total Environment 569-570: $1232-1240$.

DOI: https://doi.org/10.1016/j.scitotenv.2016.06.198

Hernández ME. 2016. Las bacterias metanotróficas y desnitrificantes en humedales de agua dulce en México: sus implicaciones en los servicios ambientales. En: Arena-Ortiz ML, Chiappa-Carrara X. comps. Microbiologia Ambiental en México. Yucatan, México: Universidad Nacional Autónoma de México, 82-92. ISBN: 978-607-02-9617-8

Jackson MB, Colmer TD. 2005. Response and adaptation by plants to flooding stress. Annals of Botany 96: 501-505. DOI: https://doi.org/10.1093/aob/mci205

Keeley JE. 1979. Population differentiation along a flood frequency gradient: physiological adaptations to flooding in Nyssa sylvatica. Ecological Monographs 49: 89-108. DOI: https://doi.org/10.2307/1942574

Kludze H, DeLAune RD, Patrick WH 1994. A colorimetric method for assaying dissolved oxygen loss from containergrown rice roots. Agronomy Journal 86: 483-487. ISSN: 0002-1962

Lai W, Zhang Y, Chen Z. 2012. Radial oxygen loss, photosynthesis, and nutrient removal of 35 wetland plants. Ecological Engineering 39: 24-30.

DOI: https://doi.org/10.1016/j.ecoleng.2011.11.010

Lemoine DG, Mermillod-Blondin F. 2012. The ability of aquatic macrophytes to increase root porosity and radial oxygen loss determines their resistance to sediment anoxia. Aquatic Ecology 46: 191-200.

DOI: https://doi.org/10.1007/s10452-012-9391-2

Malvárez IA, Fabián R. 2004. Bases ecológicas para la clasificación e inventario de humedales en Argentina. Buenos Aires, Argentina: ISBN: 9872157502

Matsui T, Tsuchiya T. 2006. A method to estimate practical radial oxygen loss of wetland plant roots. Plant and Soil 279: $119-128$.

DOI: https://doi.org/10.1007/s11104-005-0462-1

Matsui T, Tsuchiya T. 2008. Interspecific differences in radial 
oxygen loss from the roots of three Typha species. Limnology. 9: 207-211 DOI:

https://doi.org/10.1007/s10201-008-0253-5

Mei XQ, Ye ZH, Wong MH. 2009. The relationship of root porosity and radial oxygen loss on arsenic tolerance and uptake in rice grains and straw. Environmental Pollution 157: 25502557. DOI: https://doi.org/10.1016/j.envpol.2009.02.037

Mitsch WJ, Gosselink JG. 2015. Wetlands. Wiley. ISBN: 9781-118-67682-0

Travieso-Bello AC, Moreno-Casasola P, Campos A. 2005. Efecto de diferentes manejos pecuarios sobre el suelo y la vegetación en humedales transformados a pastizales. Interciencia 30: 12-18.

Moreno-Casasola P, Cejudo-Espinosa E, Capistrán-Barradas A, Infante-Mata D, López-Rosas H, Castillo-Campos G, PalePale J, Campos-Cascaredo A. 2010. Composición florística, diversidad y ecología de humedales herbáceos emergentes en la planicie costera central de Veracruz. Boletín de la sociedad botánica de México 87: 29-50.

DOI: http://dx.doi.org/10.17129/botsci.291

Sasikala S, Tanaka, Wah HSY, Jinadasa KBSN. 2009. Effects of water level fluctuation on radial oxygen loss, root porosity, and nitrogen removal in subsurface vertical flow wetland mesocosms. Ecological Engineering 35: 410-417. DOI: https://doi.org/10.1016/j.ecoleng.2008.10.003

Sorrell BK. 1999. Effect of external oxygen demand on radial oxygen loss by Juncus roots in titanium citrate solutions. Plant, Cell and Environment 22: 1587-1593.

DOI: https://doi.org/10.1046/j.1365-3040.1999.00517.x

Van-Noordwijk M, Brouwer G. 1988. Quantification of airfilled root porosity: A comparison of two methods. Plant and Soil, 111: 255-258.

DOI: https://doi.org/10.1007/978-94-009-0891-8_41

Wiessner A, Kuschk P, Stottmeister U. 2002. Oxygen Release by Roots of Typha latifolia and Juncus effuses in Laboratory Hydroponic Systems. Acta Biotechnol. 22: 209-216.

Zhang J, Wu H, Hu Z, Liang S, Fan J. 2014. Examination of oxygen release from plants in constructed wetlands in different stages of wetland plant life cycle. Environmental Science and Pollution Research, 21: 9709-9716. DOI: https://doi.org/10.1007/s11356-014-2905-9

Editor de sección: Enrique Jurado

Contribución de autores: ESO: Ejecutó las mediciones, proceso datos, elaboró gráficas y análisis estadísticos. JLMM: Participo en la redacción y edición del manuscrito. MEHA: Proporcionó la idea del proyecto, proporcionó el financiamiento e infraestructura para realizarlo, redactó el manuscrito, analizó y discutió los resultados. 